\title{
GW optical counterpart search in the Multi-Messenger Astronomy Era
}

\author{
A. Grado*1, E. Brocato ${ }^{2}$, M. Branchesi ${ }^{3}$, E. Cappellaro ${ }^{4}$, S. Covino ${ }^{5}$, F. Getman ${ }^{1}$, G.

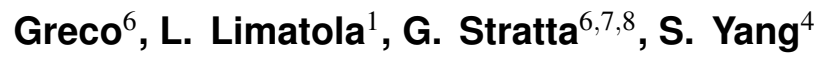

on behalf of the larger GRAWITA collaboration

${ }^{1}$ INAF, Osservatorio Astronomico di Capodimonte, salita Moiariello 16, I-80131, Napoli, Italy

E-mail: aniello.grado@.inaf.it

${ }^{2}$ INAF, Osservatorio Astronomico di Roma, Via di Frascati, 33, I-00078 Monteporzio Catone, Italy

${ }^{3}$ Gran Sasso Science Institute, Viale F. Crispi, 767100 L'Aquila, Italy

${ }^{4}$ INAF, Osservatorio Astronomico di Padova, Vicolo dell'Osservatorio 5, I-35122 Padova, Italy

${ }^{5}$ INAF, Osservatorio Astronomico di Brera, Via E. Bianchi 46, I-23807 Merate (LC), Italy

${ }^{6}$ Università degli Studi di Urbino 'Carlo Bo', Dipartimento di Scienze Pure e Applicate, P.za

Repubblica 13, I-61029, Urbino, Italy

${ }^{7}$ INFN, Sezione di Firenze, I-50019 Sesto Fiorentino, Firenze, Italy

${ }^{8}$ INAF-OAS Bologna, via Gobetti 93, 40129, Bologna, Italy

\begin{abstract}
We report on the deep optical follow-up surveys of the four gravitational-wave events accomplished by the GRAvitational Wave Inaf TeAm Collaboration (GRAWITA) using the VLT Survey Telescope (VST). We responded promptly to the gravitational-wave alerts sent by the LIGO and Virgo Collaborations, covering a region of $90 \mathrm{deg}^{2}, 72 \mathrm{deg}^{2}$ and $99 \mathrm{deg}^{2}$ for GW150914, GW151226 and GW170814 respectively, and kept observing the three areas over nearly two months. The surveys average limiting magnitude reached about 22 mag AB in the $r$-band. For GW170817 event we secured deep observations of AT2017gfo's host galaxy NGC4993. The paper outlines the VST observational strategy and two independent procedures developed to search for transient counterpart candidates in multi-epoch VST images. For BBH events, numerous transients have been discovered, mostly variable stars and eclipsing binaries, but no candidates are identified as related to the gravitational-wave events. For GW170817 event the VST observations allows to measure the optical transient magnitudes at the faint end of the light curve.
\end{abstract}

GRAvitational-waves Sciencetechnology Symposium - GRASS2018

1-2 March 2018

Palazzo Moroni, Padova (Italy)

\footnotetext{
* Speaker.
} 


\section{Introduction}

Gravitational waves (GWs) are perturbations of space-time metric produced by a time dependent mass quadrupole moment. GWs are generated by different kinds of astrophysical sources. Among those, coalescence of binary systems of compact objects such as neutron stars (BNS), NS and a stellar-mass black hole (NSBH) or black holes (BBH), collapse of massive stars with large degree of asymmetry and fast rotating asymmetric isolated NSs.

In September 2015, the detection of a gravitational wave was finally achieved with the detection by the LIGO and Virgo Collaboration (LVC) of unambiguous emission of GW radiation from an astrophysical source. After a long and detailed analysis, it was recognized that the event was originated by the coalescence of two BHs at a cosmological redshift of $z \simeq 0.09$ [2]. Since then further five detections plus one candidate event have been announced by LVC ${ }^{1}$. The sky localization of the GW sources, obtained through triangulation with the two aLIGO interferometers located in Hanford (Washington) (H1) and Livingston (Louisiana) (L1) [1] and aVirgo [3], spans from a few tents to thousand of square degrees [16, 10]. For the last event GW170817 generated by the coalescence of two binary neutron stars, the contribution of aVirgo allows to reduce the $90 \%$ sky credible region to $28 \mathrm{deg}^{2}$ allowing the identification of the electromagnetic (EM) counterpart.

In this paper we describe the observational campaign performed by the GRAvitational Wave INAF TeAm (GRAWITA) ${ }^{2}$ to follow up four of the GW triggers during the $\mathrm{O} 1$ and $\mathrm{O} 2$ LVC scientific run by using the ESO-VLT Survey Telescope (VST), its results and the prospects for the upcoming years. In section 2 some details on the VST telescope and the observational strategy are presented, including the specific observational response to the LVC triggers. A brief summary of the adopted pre-reduction is described in section 3. In the same section, we present our approach to the transient search and introduce the two independent pipelines we developed to this aim. In the following section 4, the results of the search are described. A brief discussion close the paper (section 5).

\section{VST observational strategy}

GW sky localization is relatively poor, this represents a challenge for the identification of electromagnetic transients associated to GW signals. Depending on the distance of the source and localization error area two approaches can be followed to optimize the search. In case of nearby GW source $(<100 \mathrm{Mpc})$ and even moderately small localization area the most efficient approach is to image each galaxy within the error volume separately. This approach is very effective in narrowing the search area. On the other side if the search volume starts to be wide, the number of galaxies becomes too high and a blind search covering the whole error area is more effective. The VLT Survey Telescope (VST) for his characteristics of field of view, aperture and spatial resolution fits well the second approach. VST is a $2.6 \mathrm{~m}, 1 \mathrm{deg}^{2}$ field of view (FoV) imaging telescope located at the Cerro Paranal Observatory in Chile [7] and dedicated to large sky surveys in the austral hemisphere. The telescope optical design allows to achieve an uniform PSF with variation $<10 \%$

\footnotetext{
${ }^{1}$ http://chrisnorth.github.io/plotgw/

${ }^{2}$ https://www.grawita.inaf.it/
} 
Table 1: Log of the VST observations for the GW events. The latency is the time between the start of the observations and the GW event alert, the coverage of the initial sky map is the fraction in percentage of the initial pycbc/bayestar $90 \%$ localization area covered with the VST. The coverage of the refined sky map is the fraction in percentage of the final LALInference $90 \%$ localization area covered with the VST. In column six are reported the number of epochs observed for the specified event and the last column report the 50\% completeness for point like sources.

\begin{tabular}{lcccccc}
\hline \hline Event & $\begin{array}{c}\text { Latency } \\
\text { (hours) }\end{array}$ & $\begin{array}{c}\text { Filter } \\
-\end{array}$ & $\begin{array}{c}\text { Coverage } \\
\text { Initial sky } \\
\text { map (\%) }\end{array}$ & $\begin{array}{c}\text { Coverage } \\
\text { refined sky } \\
\text { map (\%) }\end{array}$ & $\begin{array}{c}\text { \# of epochs } \\
-\end{array}$ & $\begin{array}{c}\text { Completeness } \\
\text { (AB mag) }\end{array}$ \\
\hline GW150914 & 23 & $\mathrm{r}$ & 29 & 10 & 6 & 21 \\
GW151226 & 7.6 & $\mathrm{r}$ & 9 & 7 & 6 & 21 \\
GW170814 & 17.5 & $\mathrm{r}$ & 77 & 54 & 6 & 22.5 \\
GW170817 & 9.15 & $\mathrm{r}$ & 31 & 15 & 1 & 22.5 \\
NGC4993 & $5.4 \mathrm{~d}$ & $\mathrm{~g}, \mathrm{r}, \mathrm{i}, \mathrm{z}$ & - & - & 1 & $23.6,23.5,22.5,21.8$ \\
NGC4993 & $14.4 \mathrm{~d}$ & $\mathrm{i}$ & - & - & 1 & 22.5 \\
NGC4993 & $145.7 \mathrm{~d}$ & $\mathrm{~g}, \mathrm{i}$ & - & - & 1 & $25.0,24.5$ \\
\hline
\end{tabular}

over the whole field of view. The VST is equipped with the OmegaCAM camera [13], which covers the field of view of 1 square degree with a scale of $0.21 \mathrm{arcsec} /$ pixel, through a mosaic of 32 CCDs.

The required time allocation for the GW follow up survey (PI A. Grado and E. Cappellaro) was obtained in the framework of the Guarantee Time Observations (GTO) assigned by ESO to the telescope and camera teams in reward of the effort for the construction of the instrument. The planned strategy of the transient survey foresees to monitor a sky area of up to $100 \mathrm{deg}^{2}$ at 5 or 6 different epochs beginning soon after the GW trigger and lasting 8-10 weeks. The typical VST Observing Block (OB), prepared using a dedicated script, publicly available, named GWsky 3 , contains groups of nine pointings (tiles) covering an area of $3 \times 3 \mathrm{deg}^{2}$. Each pointing is imaged with two exposures of $40 \mathrm{~s}$ dithered by $\sim 0.7-1.4$ arcmin to fill the OmegaCAM CCD mosaic gaps.

In table 1 is reported the summary of the observations for the four GW events followed by the VST (for details see table caption). It should be mentioned that for the event GW170817 the initial sky map issued by LVC was offset of 2.5 degrees compared to the final sky map, a remarkable result. However the VST observations, started very early, on August 17th at 23:18 UTC, were prepared on the base of the initial map. This circumstance together with the limited visibility of the area due to sun constraints, that allowed an observing time window sufficient to cover only nine $\mathrm{deg}^{2}$, result on the fact that the optical transient AT2017gfo was not included in the search area. In the next days, new observations were executed pointing the telescope on the galaxy NGC4993, as reported on the last three rows of the table 1, allowing to secure photometric measurements at the faint end of the kilonova light curve.

\footnotetext{
${ }^{3}$ https://github.com/ggreco77/GWsky
} 


\section{Data Processing}

Immediately after the observation, the images are mirrored to ESO data archive, and then transferred by an automatic procedure from ESO Headquarters to the VST Data Center in Naples (IT). The procedure also starts the image processing running VST-tube, which is the pipeline developed for the VST-OmegaCAM mosaics [11]. It includes pre-reduction, astrometric and photometric calibration and mosaic production. For further details on the data reduction see [8].

In order to search for optical transient associated to GW event, the images are analyzed using two independent procedures. One, dubbed ph-pipe, is catalogs-based and compares the photometric measurements of all the sources in the VST fields obtained at different epochs. The second, named diff-pipe, is based on the analysis of the difference of images following the approach of the supernova (SN) search program with the VST [9].

The two approaches are intended to be complementary, with the first typically faster and less sensitive to image defects and the latter more effective for sources embedded in extended objects or in case of strong crowding. For both procedures, the main goal is to identify sources showing a "significant" brightness variation (around $0.5 \mathrm{mag}$ ), either raising or declining flux, during the period of monitoring, that can be associated to extra-galactic events.

The photometric pipeline provides a list of transients in low-latency to organize immediate follow-up activities. The computation time can be particularly short, e.g. just a few minutes for each epoch surveyed by the VST. The weakness of this approach is that sources closer than about a Point Spread Function (PSF) size or embedded in extended objects can be difficult to detect and therefore can possibly remain unidentified. The diff-pipe is based on a widely used, most effective method for transients detection, i.e. the difference of images taken at different epochs [4]. To implement this approach for the survey described in this paper we exploit our experience with the medium-redshift SN search done with the VST [9]. The image difference pipeline was definitely more time consuming than the photometric pipeline, and optimization of the code is on going. For details on the implementation of the two procedures see [6]

\section{Results}

We now know that the gravitational wave events GW150914, GW151226 and GW170814, were generated by coalescence of black-holes and GW170817 was generated by the coalescence of two neutron stars. In the current scenario strong electromagnetic radiation from $\mathrm{BBH}$ is not expected to occur, and in fact none of the transients identified by the worldwide astronomical effort could be linked to the observed GW events. However, the analysis of the data obtained in response to the GW triggers is important both for evaluating the search performances and for tuning future counterpart searches. In table 2 are summarized the results found for the transients search associated to the BBH events. In the table are shown the total number of sources summed all over the epochs, then for each pipeline are reported the number of transients found after removing all the known photometric and positional transients available in SIMBAD [17] and GAIA databases [15]. The last column, as by-product, shows the total number of supernovae candidates we found in the time window of interest. The list includes the known $\mathrm{SNe}^{4}$ that were also found in our search.

\footnotetext{
${ }^{4}$ We used the update version of the Asiago SN catalog (http://sngroup.oapd.inaf.it/asnc.html) [5]
} 
Table 2: Results of the optical transients/variables search. The initial number of sources indicates the total number, summed over all the epochs, of the sources detected in the fields. The fourth columns indicates the number of transients found after removing the known photometric and positional variables sources. In the last columns, as by product, are reported the number of known and candidate supernovae found in the field.

\begin{tabular}{lcccc}
\hline \hline Event & Pipeline & $\begin{array}{c}\text { Initial number } \\
\text { of sources }\end{array}$ & optical transients & $\mathrm{SNe}$ \\
\hline GW150914 & ph-diff & $9 \times 10^{6}$ & 1300 & 10 \\
& ph-pipe & & 939 & - \\
GW151226 & ph-diff & $9 \times 10^{5}$ & 1113 & 21 \\
& ph-pipe & & 305 & - \\
GW170814 & ph-diff & $1.5 \times 10^{6}$ & 55 & 24 \\
& ph-pipe & & 37 & \\
\hline
\end{tabular}

Table 3: Magnitudes of the AT2017gfo measured with the VST. The modified Julian day (MJD) is defined as JD-2,400,000.5. In the first three rows of the last column are reported the transient AB magnitudes. For the last four observations, below the horizontal line, the OT was not detected and the column reports the 50 $\%$ completeness limit for point like sources. The typical error associated to the these values are around 0.2 mag

\begin{tabular}{lccc}
\hline \hline MJD & Filter & Exptime (s) & mag (AB) \\
\hline 57987.99 & $\mathrm{~g}$ & 200 & $22.51 \pm 0.12$ \\
57988.98 & $\mathrm{r}$ & 200 & $21.31 \pm 0.07$ \\
57988.97 & $\mathrm{i}$ & 200 & $20.33 \pm 0.09$ \\
\hline 57988.97 & $\mathrm{z}$ & 200 & 21.8 \\
57996.97 & $\mathrm{i}$ & 1500 & 23.3 \\
58123.29 & $\mathrm{~g}$ & 5400 & 25.01 \\
58123.32 & $\mathrm{r}$ & 5400 & 24.55 \\
\hline
\end{tabular}

A comparison between the transients identified by the two pipelines shows that, as expected, the image-difference pipeline is more effective, in particular for objects very close to extended sources. However, the photometric pipeline is less affected by image defects as halos of very bright or saturated stars. For the GW170817 event, we measured the magnitudes of the AT2017gfo transient as reported in table 3 [14]. For observations starting from August 31st, the transient was not visible in the images, so in the table are reported the $50 \%$ completeness to be considered as upper limits to transient magnitudes.

\section{Summary.}

In this proceeding we report on the GRAWITA contribution to the search of the optical counterparts for the GW events, GW150914, GW151226, GW170814 and GW170817, exploiting the capabilities of the VLT survey telescope. For GW produced by BBH coalescence none of the transients identified by our team can be related to the gravitational events. Nevertheless, this work 
made possible to verify the capabilities, reliability and the effectiveness of our project. We covered a search area up to $99 \mathrm{deg}^{2}$ repeated in six epochs distributed typically over 2 months. A $50 \%$ point source completeness of the order of $r \simeq 22 \mathrm{mag}$ was reached in most of the epochs. For the search of optical transients two independent pipelines have been developed. One based on source extraction and magnitude comparison between different epochs and the second on transient identification obtained through image subtraction techniques. The two pipelines allow the identification of a number of astrophysical transients, none of them can be related to the gravitational event. For the BNS event GW170817 the VST measured the AT2017gfo transient magnitude in g,r,i filters 5.5 days after the detection. Further observations were conducted in the next days, only upper limits to the magnitudes were put due to the faintness of the source. We plan to devote around 80 hours of VST observing time per semester in the next three years for the search of GW optical counterparts. Depending on the surveyed area size and assuming six epochs for event this will allow us to follow up to eight GWs for semester.

\section{6. acknowledgments}

This paper is based on observations made with the ESO/VST. We acknowledge the usage of the VST Italian GTO time. We also acknowledge INAF financial support of the project "Gravitational Wave Astronomy with the first detections of adLIGO and adVIRGO experiments". Facility: VST ESO programs: 095.D-0195, 095.D-0079 and 096.D-0110, 096.D-0141, 099.D-0191, 099.D-0568, 0100.D-0022.

\section{References}

[1] Aasi, J., Abbott, B. P., et al. 2015, Classical and Quantum Gravity, 32, 074001

[2] Abbott, B. P., Abbott, R., Abbott, T. D., et al. 2016a, Physical Review Letters, 116, 061102

[3] Acernese, F., Agathos, M., Agatsuma, K. et al. 2015 Classical and Quantum Gravity, 32, 2, 024001

[4] Alard, C. 1999, arXiv:9903111

[5] Barbon R., Buondí V., Cappellaro E., Turatto M., 1999, A\&AS, 139, 531

[6] Brocato, E., Branchesi, M., Cappellaro, E., et al. 2018 MNRAS, 474, Issue 1, 411

[7] Capaccioli, M., Mancini D., Sedmak, G. 2003, MmSAI, 74, 450

[8] Capaccioli, M., Spavone, M., Grado, A. et al. 2015, A\&A, 581, 10

[9] Cappellaro, E., Botticella, M.T., Pignata, G. et al. 2015, A\&A, 584, 62

[10] Essick, R., Vitale, S., Katsavounidis, E., Vedovato, G., \& Klimenko, S. 2015, ApJ, 800, 81

[11] Grado, A. et al. 2012, MSAIS, 19, 362

[12] Klimenko, S., Vedovato, G., Drago, M., et al. 2016, PhyRevD, 93, 4, 042004

[13] Kuijken, K. 2011, The Messenger, 146, 8

[14] Pian E., D’ Avanzo P., Benetti, S. et al 2017, Nature, 551, 7678, 67

[15] Salgado, J., et al., 2017, A\&C, 21, 22

[16] Singer, L. P., Price, L. R., Farr, B., et al. 2014, ApJ, 795, 105

[17] Wenger, M. et al. 2000, A\&AS, 143, 9 